\title{
Transcranial direct current stimulation reduces post-ERCP pain
}

Transcranial direct current stimulation (tDCS) can reduce pain following endoscopic retrograde cholangiopancreatography (ERCP), according to the results of a recent study by Borckardt et al. published in Gastrointestinal Endoscopy.

Evidence is emerging for the potential use of various novel brain stimulation technologies-including transcranial magnetic stimulation (TMS) and tDCSfor the treatment and management of pain. "Electricity has no metabolite or other residue and can be delivered with minimal to no discomfort," the authors of the study write.

Patients undergoing ERCP can experience severe pain both during and after the procedure, which can result in the need to be kept in hospital overnight. Borckardt et al., therefore, decided to investigate the feasibility, safety and effectiveness of tDCS for reducing postERCP pain. The advantages of $\mathrm{tDCS}$ over TMS include the fact that tDCS is less complicated to deliver, less

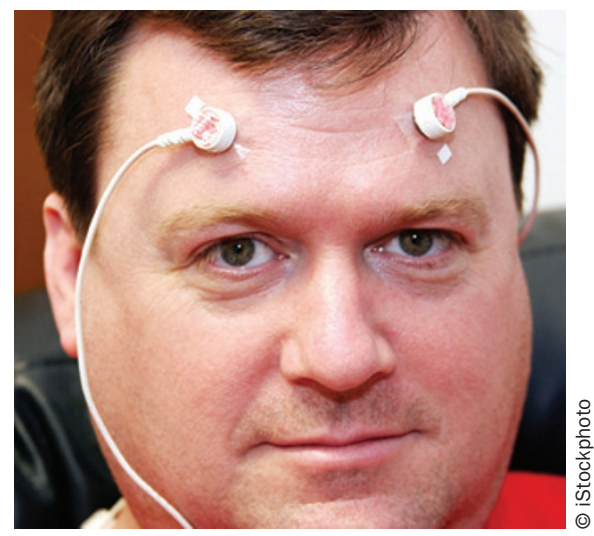

cumbersome and is associated with fewer adverse effects.

21 patients who were scheduled to undergo ERCP were enrolled in the study. Patients were randomly allocated to receive 20 min of either real or sham tDCS immediately after ERCP. Pain was measured using a visual analogue scale (VAS), the McGill pain questionnaire and the brief pain inventory. Patientcontrolled analgesia use and adverse events were also recorded.
Patients who received real tDCs used $22 \%$ less total hydromorphone than those who received sham tDCS. "Despite using less self-administered opioid medication, participants in the real tDCS group reported less pain and better mood across all VAS measures used (pain on average, pain at its worst, pain unpleasantness, bothersome pain, and mood)," the researchers report. There were no serious adverse effects associated with tDCS; such effects were limited to tingling, itching and mild stinging under the electrodes.

"The role of $\mathrm{tDCS}$ in pain management is not yet well-established; the optimal cortical targets, dosing strategies, electrode configurations, and electrode types also need further study," the authors conclude.

Isobel Franks

Original article Borckardt, J. J. et al. Feasibility, safety, and effectiveness of transcranial direct current stimulation for decreasing post-ERCP pain: a randomized, shamcontrolled, pilot study. Gastrointest. Endosc. doi:10.1016/ j.gie.2011.01.050 